\title{
Hair Morphology of Striped Hyena Hyaena hyaena (Linnaeus, 1758)
}

\author{
Manokaran Kamalakannan ${ }^{1}$ and Joy Krishna De $^{2}$ \\ ${ }^{1}$ Zoological Survey of India, M- Block, New Alipore, Kolkata-700053, India \\ ${ }^{2}$ West Bengal Zoo Authority, KB-19, Salt Lake, Sector-III, Kolkata-700098, India \\ *Corresponding author
}

\begin{abstract}
A B S T R A C T
Keywords

Hyaena hyaena, Hair morphology, Dorsal guard hair.

Article Info

Accepted:

17 April 2017

Available Online:

10 May 2017

The microscopic characteristics of dorsal guard hairs of Hyaena hyaena were examined using optical light microscope to know its hair morphology. The hair of $H$. hyaena is diagnosed by its unique medullary characteristics i.e. the composition of medulla- 'unicellular regular', structure of medulla'simple or narrow medulla lattice' and the 'oblong' shape cross-section. The photo-micrographs and characteristics of dorsal guard hairs are presented here may be used for the species identification.
\end{abstract}

\section{Introduction}

Hair morphology is adapted to identify the mammal's species when the external morphology is unable to provide a proper result for identifying the mammal's species (Teerink, 1991; Chakraborty and De, 2010). The physical and microscopic characteristics of the Indian mammals have been well documented by many authors (Bahuguna et al., 2010; Chakraborty and De, 2010; Sarkar et al., 2011; Kamalakannan et al., 2013; Kamalakannan, 2017a and b). The different orders of class Mammalia were dealt by De, 1993; Primates; Chakraborty and De, 2010; Carnivora; Bahuguna et al., 2010; selected mammals; Sarkar, 2012; Rodentia; Kamalakannan, 2015, Artiodactyla and Lagomorpha). However, scanty information is available on the hair structure of Hyaena hyaena. Therefore, the present study was undertaken to provide a detailed microscopic characteristics of hairs of $H$. hyaena for its species identification.

The striped hyena is a large dog-like carnivore placed under separate family Hyaenidae; its pelage is long and the coat colour is tawny-yellow infused with broad vertical black stripes on the flanks, and fore and hind legs; heavy crest of long hairs over the neck and back; in India this animal is distributed in south to the Nilgiri hills, west to Gujarat, north to lowland of Jammu and Kashmir and Kumaon, east to West Bengal; habitat loss and degradation, poisoning and 
poaching are the major threats to this species; conservation status of this species is as per the IUCN Red List- Near Threatened; Indian Wildlife (Protection) Act, 1972- Schedule III; CITES- Appendix- III (Menon, 2014).

\section{Materials and Methods}

About 10-15 tufts of dorsal guard hair were collected from the mid-dorsal region of dry specimens of $H$. hyaena present in the National Zoological Collection, Mammal and Osteology Section of the Zoological Survey of India, Kolkata, India. The samples were washed thoroughly in carbon tetra chloride after by Chakraborty et al., (1996) to remove the dirt of exogenous materials. Microscopic characters such as scale position, scale pattern, scale margin and scale margin distance of dorsal guard hair were studied with help of the digital camera fitted on an optical light microscope (Olympus BX41).

The medullary configuration and composition, structure and margins of the medulla and cross-section of dorsal guard hair were recorded and photographed. Nomenclature of different parameters was followed by Bruner and Coman (1974); Moore et al., (1974); Teerink (1991) and Chakraborty et al., (1996).

\section{Results and Discussion}

The cuticular characteristics of dorsal guard hair of $H$. hyaena were observed as: scale position- 'transversal', scale patterns- 'regular wave', the structure of scale margins'smooth' and the distance between scale margins- 'near'. The medullary characteristics of dorsal guard hair were as: composition of medulla- 'unicellular regular', the structure of medulla- 'simple or narrow medulla lattice', and form of the medulla margins- 'straight'. The cross-section of dorsal guard hair was observed as 'oblong' shape (Table 1; Figure 1).

The hair of $H$. hyaena can be identified with its unique medullary characteristics i.e. the composition of medulla- 'unicellular regular', structure of medulla- 'simple or narrow medulla lattice' and the 'oblong' shape crosssection. Although the cuticular characteristics are similar between other mammals species were studied earlier by Chakraborty and De (2010); Sarkar (2012); Kamalakannan (2015), the cuticular characters can be used as a combination of characters. There is no specific study on hair morphology of $H$. hyaena except a study Chakraborty and De (2010), in which, the surface structure of $H$. hyaena was discussed.

Table.1 Microscopic hair characteristics of Hyaena hyaena

\begin{tabular}{|l|l|}
\hline \multicolumn{2}{|c|}{ a. Cuticular scale characteristics } \\
\hline Scale position & Transversal \\
\hline Scale patterns & Regular wave \\
\hline Structure of scale margins & Smooth \\
\hline Distance between scale margins & Near \\
\hline \multicolumn{2}{|c|}{ b. Medullary characteristics } \\
\hline Composition of medulla & Unicellular regular \\
\hline Structure of medulla & $\begin{array}{l}\text { Simple / } \\
\text { Narrow medulla lattice }\end{array}$ \\
\hline Margins of medulla & Straight \\
\hline c. Shape of cross-section & Oblong \\
\hline
\end{tabular}


Fig.1 Photo-micrograph of dorsal guard hair characteristics of Hyaena hyaena

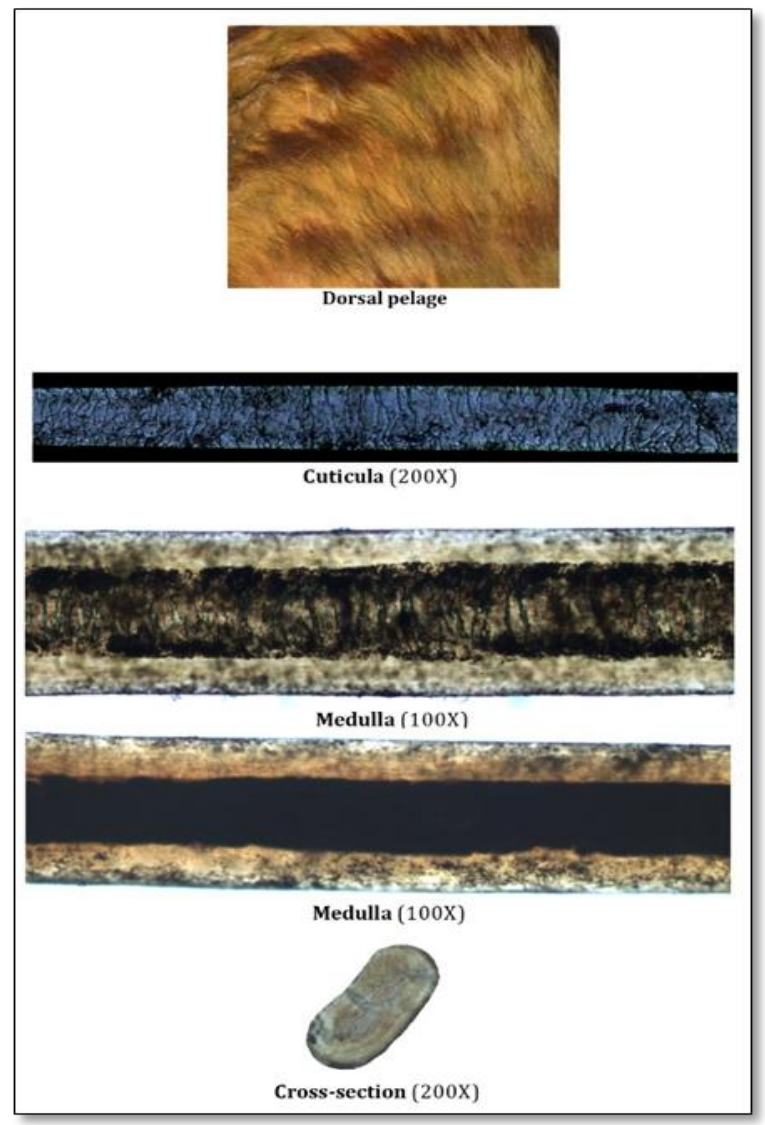

The cuticular characters, medullary configuration and cross-section of dorsal guard hair are more or less similar to observation of Chakraborty and De (2010). However, this study provides a complete combination of characters of dorsal guard hair of $H$. hyaena along with the high-resolution photo-micrographs for species identification.

\section{Acknowledgements}

The authors sincerely thank the Director, Zoological Survey of India, Kolkata for providing necessary facilities and encouragements.

\section{References}

Bahuguna, A., Sahajpal, V., Goyal, S.P., Mukherjee, S.K. and Thakur, V. 2010.
$\mathrm{CD}$ version of forensic guide for identification of selected mammals from primary guard hair. Wildlife Institute of India, Dehradun 438 pp.

Brunner, H. and Comman, B. 1974. The Structure of Hairs. Inkata Press, Melbourne, Australia, 170 pp.

Chakraborty, R., De, J.K. and Chakraborty, S. 1996. Identification of dorsal guard hairs of Indian species of the genus Panthera Oken (Carnivora: Felidae). Mammalia, 60: 473-480.

Chakraborty, R. and De, J.K. 2010. Atlas on hairs Indian Mammals Part- I: Carnivora: Published by the Director, Zoological Survey India, $141 \mathrm{pp}$.

De, J.K. 1993. Study of surface structure of hair of some Primates of Indian subcontinent. Records of the Zool. Survey of India, 93: 31-34. 
Kamalakannan, M., De, J.K. and Manna, C.K. 2013. Identification of dorsal guard hairs surface structure of Indian chevrotain Moschiola Indica Gray, 1852 (Tragulidae: Artiodactyla: Mammalia). Biolife, 1(3): 155-158.

Kamalakannan, M. 2015. Tricho-taxonomic studies of Indian mammal species belonging to the orders Artiodactyla and Lagomorpha. Ph.D thesis. University of Kalyani, West Bengal, India.

Kamalakannan, M. 2017. Identification of hairs of Ratufa bicolor (Sparrman, 1778), Ratufa indica (Erxleben, 1777) and Ratufa macroura Pennant, 1769. J. Entomol. Zool. Studies, 5(2): 983-985.

Kamalakannan, M. 2017. Characterisations of hair of Hoolock Gibbon Hoolock hoolock (Harlan, 1834) (Hylobatidae: Primates: Mammalia). J. Entomol. Zool. Studies, 5(2): 986-988.

Menon, V. 2014. Indian mammals- a field guide, Hachette (India) Pvt. Limited. $528 \mathrm{pp}$.

Moore, T.D., Spence, L.E and Dugnolle, C.E. 1974. Identification of the dorsal guard hairs of some mammals of Wyoming. Game and Fish Department, Wyoming, $177 \mathrm{pp}$.

Sarkar, P.S., De, J.K. and Manna, C.K. 2011. Identification of dorsal guard hair of five species of the family Cercopithecidae (Primates: Mammalia). Curr. Sci., 100: 1725-1728.

Sarkar P.S. 2012. Tricho-taxonomic study of hairs of Indian mammals: orderPrimates and Rodentia, Ph.D thesis, University of Kalyani, Kalyani, West Bengal, India.

Teerink, B.J. 1991. Hair of West-European mammals atlas and identification key. Cambridge: Cambridge University Press, 223 pp.

\section{How to cite this article:}

Manokaran Kamalakannan and Joy Krishna De. 2017. Hair Morphology of Striped Hyena Hyaena hyaena (Linnaeus, 1758). Int.J.Curr.Microbiol.App.Sci. 6(5): 1438-1441. doi: https://doi.org/10.20546/ijcmas.2017.605.157 